\title{
AN AVERAGING FORMULA FOR THE COINCIDENCE REIDEMEISTER TRACE
}

\author{
JONG BUM LEE AND P. CHRISTOPHER STAECKER
}

\begin{abstract}
In the setting of continuous maps between compact orientable manifolds of the same dimension, there is a well known averaging formula for the coincidence Lefschetz number in terms of the Lefschetz numbers of lifts to some finite covering space. We state and prove an analogous averaging formula for the coincidence Reidemeister trace. This generalizes a recent formula in fixed point theory by Liu and Zhao.

We give two separate and independent proofs of our main result: one using methods developed by Kim and the first author for averaging Nielsen numbers, and one using an axiomatic approach for the local Reidemeister trace.

We also give some examples and state some open questions for the nonorientable case.
\end{abstract}

\section{CONTEnTs}

1. The Reidemeister trace in fixed point theory 1

2. Coincidence theory 6

3. Main result 9

4. An axiomatic proof 10

5. Examples 17

6. Open questions in the nonorientable case 20

References 22

\section{The Reidemeister trace in FiXed POINT theory}

Let $X$ be a compact polyhedron with the universal covering projection $p: \tilde{X} \rightarrow X$ and let $\Pi$ be the group of all covering transformations of $p$. Let $f: X \rightarrow X$ be a self-map and $\tilde{f}: \tilde{X} \rightarrow \tilde{X}$ be a lift of $f$. We define an endomorphism $\phi: \Pi \rightarrow \Pi$ given by the rule

$$
\phi(\alpha) \tilde{f}=\tilde{f} \alpha, \quad \forall \alpha \in \Pi .
$$

2010 Mathematics Subject Classification. 54H25, 55M20.

Key words and phrases. Averaging formula, coincidence point, fixed point, Nielsen theory, Reidemeister trace.

The first author is supported by Basic Science Researcher Program through the National Research Foundation of Korea (NRF-2016R1D1A1B01006971). 
Let $\mathcal{R}[\phi]$ denote the set of Reidemeister classes of $\phi$, defined by the twisted conjugacy relation $\alpha \sim \beta$ if and only if there is some $\gamma$ with $\alpha=\gamma^{-1} \beta \phi(\gamma)$. We write $[\alpha]_{\phi}$ for the Reidmeister class of $\alpha$. When the $\phi$ is understood, we simply write $[\alpha]$.

We can consider the free Abelian group $\mathbb{Z} \mathcal{R}[\phi]$ with generators given by the Reidemeister classes. There is a one-to-one correspondence between the Nielsen fixed point classes $\mathbb{F}$ and the Reidemeister classes $[\alpha]$. Thus the Nielsen classes $\mathbb{F}$ can be indexed by the Reidemeister classes $[\alpha] ; \mathbb{F}=\mathbb{F}_{[\alpha]}$.

The Reidemeister number $R(f)=R(\phi)$ is the number of Reidemeister classes. The Lefschetz number $L(f)$ defined using the homology groups is the sum of local indices, which is an important result obtained by Lefschetz. That is,

$$
L(f)=\sum_{[\alpha] \in \mathcal{R}[\phi]} \operatorname{ind}\left(f, \mathbb{F}_{[\alpha]}\right) \in \mathbb{Z} .
$$

The Reidemeister trace of $f$ is defined as follows:

$$
R T(f, \tilde{f})=\sum_{[\alpha] \in \mathcal{R}[\phi]} \operatorname{ind}\left(f, \mathbb{F}_{[\alpha]}\right)[\alpha] \in \mathbb{Z} \mathcal{R}[\phi] .
$$

The Reidemeister trace contains information of the Nielsen number and the Lefschetz number. Indeed, the number of non-zero terms in this sum is the Nielsen number $N(f)$, and the sum of the coefficients is the Lefschetz number $L(f)$.

Let $\Gamma$ be a finite index normal subgroup of $\Pi$ which is $\phi$-invariant. Letting $\bar{\Pi}=\Pi / \Gamma$, we have the following commutative diagram

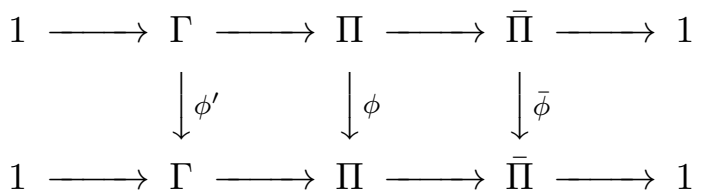

Let $\bar{X}=\Gamma \backslash \tilde{X}, p^{\prime}: \tilde{X} \rightarrow \bar{X}$ and $\bar{p}: \bar{X} \rightarrow X$. Then we have the following commutative diagram

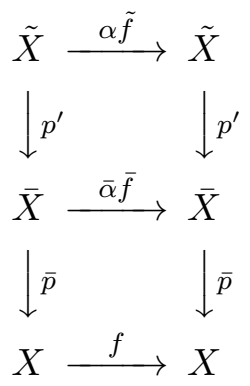

where $\alpha \in \Pi$ and $\bar{\alpha}$ is the image of $\alpha$ in $\bar{\Pi}$. Note that $\{\alpha \tilde{f} \mid \alpha \in \Pi\}$ is the set of all lifts on $\tilde{X}$ of $f$, and $\{\bar{\alpha} \bar{f} \mid \alpha \in \Pi\}$ is the set of all lifts on $\bar{X}$ of $f$. 
It has been known for some time that in this setting the Lefschetz number obeys the following averaging formula:

$$
L(f)=\frac{1}{[\Pi: \Gamma]} \sum_{\bar{\beta} \in \bar{\Pi}} L(\bar{\beta} \bar{f}) .
$$

This formula is apparently due to Jiang, and appears in [6] from 1983. The averaging formula is interesting in its own right, but also computationally useful. When the finite covering space $\bar{X}$ is topologically simpler than $X$ itself, the individual terms $L(\bar{\beta} \bar{f})$ may be easy to compute even when $L(f)$ itself is hard to compute.

It is natural to ask if a similar averaging formula holds for the Nielsen number $N(f)$. This is the subject of [7], which shows that the corresponding formula for $N(f)$ does not hold in general.

Recall from (1-1) that the endomorphism $\phi$ was defined using the lift $\tilde{f}$. Taking projection by $p^{\prime}$ we have

$$
\bar{\phi}(\bar{\alpha}) \bar{f}=\bar{f} \bar{\alpha}, \quad \forall \alpha \in \Pi .
$$

If we use other lifts $\alpha \tilde{f}$ and $\bar{\alpha} \bar{f}$ of $f$, then we obtain the corresponding endomorphisms $\tau_{\alpha} \phi$ and $\tau_{\bar{\alpha}} \bar{\phi}$ respectively. (Here, $\tau_{\alpha}$ is the conjugation by $\alpha$, $\beta \mapsto \alpha \beta \alpha^{-1}$.) Similarly, the lift $\alpha \tilde{f}$ of $\bar{\alpha} \bar{f}: \bar{X} \rightarrow \bar{X}$ induces an endomorphism $\tau_{\alpha} \phi^{\prime}: \Gamma \rightarrow \Gamma$. Hence

$$
R T(\bar{\alpha} \bar{f}, \alpha \tilde{f})=\sum_{[\gamma]_{\tau_{\alpha} \phi^{\prime}} \in \mathcal{R}\left[\tau_{\alpha} \phi^{\prime}\right]} \operatorname{ind}\left(\bar{\alpha} \bar{f} ; \mathbb{F}_{[\gamma]_{\tau_{\alpha} \phi^{\prime}}}\right)[\gamma]_{\tau_{\alpha} \phi^{\prime}},
$$

where ind denotes the fixed point index of a fixed point class. Furthermore, we have for any $\alpha \in \Pi$,

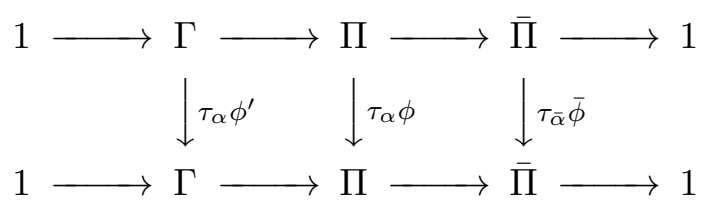

The diagram induces the following short exact sequence of sets

$$
\mathcal{R}\left[\tau_{\alpha} \phi^{\prime}\right] \stackrel{\hat{i}^{\alpha}}{\rightarrow} \mathcal{R}\left[\tau_{\alpha} \phi\right] \stackrel{\hat{u}^{\alpha}}{\rightarrow} \mathcal{R}\left[\tau_{\bar{\alpha}} \bar{\phi}\right] \rightarrow 1
$$

and the following sequence of groups

$$
1 \rightarrow \operatorname{fix}\left(\tau_{\alpha} \phi^{\prime}\right) \rightarrow \operatorname{fix}\left(\tau_{\alpha} \phi\right) \stackrel{u_{\alpha}}{\rightarrow} \operatorname{fix}\left(\tau_{\bar{\alpha}} \bar{\phi}\right),
$$

where fix $(\phi)$ denotes the fixed subgroup of the group homomorphism $\phi$.

This exactness is used in [7] to give a convenient relabeling of the fixed point classes. Typically the fixed point classes of $f$ are labeled by the Reidemeister classes $\mathcal{R}[\phi]$ of $\phi$. We may instead relabel them in terms of the Reidemeister classes $\mathcal{R}[\bar{\phi}]$ and $\mathcal{R}\left[\tau_{\alpha} \phi^{\prime}\right]$. This gives the following: 
Lemma 1.1 ([7, Lemma 2.2]). For each $[\bar{\beta}] \in \mathcal{R}[\bar{\phi}]$, choose some $[\beta] \in \mathcal{R}[\phi]$ with $\hat{u}^{1}([\beta])=[\bar{\beta}]$. Then we have the disjoint union:

$$
\operatorname{Fix}(f)=\bigsqcup_{[\bar{\beta}]_{\bar{\phi}} \in \mathcal{R}[\bar{\phi}][\gamma]_{\tau_{\beta} \phi} \in \operatorname{im}\left(\hat{i}^{\beta}\right)} p(\operatorname{Fix}(\gamma \beta \tilde{f})) .
$$

Remark 1.2. It will be important for us to relate the quantities $R T(f, \tilde{f}) \in$ $\mathcal{R}[\phi]$ and $R T(f, \alpha \tilde{f}) \in \mathcal{R}\left[\tau_{\alpha} \phi\right]$ for some $\alpha \in \Pi$. By (1-1), we have that

$$
R T(f, \alpha \tilde{f})=\sum_{[\beta]_{\tau_{\alpha} \phi} \in \mathcal{R}\left[\tau_{\alpha} \phi\right]} \operatorname{ind}\left(f ; \mathbb{F}_{[\beta]_{\tau_{\alpha} \phi}}\right)[\beta]_{\tau_{\alpha} \phi} .
$$

In this sum, we note that

$$
\mathbb{F}_{[\beta]_{\tau_{\alpha} \phi}}=p(\operatorname{Fix}(\beta(\alpha \tilde{f})))=p(\operatorname{Fix}((\beta \alpha) \tilde{f}))=\mathbb{F}_{[\beta \alpha]_{\phi}}
$$

and that multiplication on the right by $\alpha$ gives a one-to-one correspondence $\rho_{\alpha}: \mathcal{R}\left[\tau_{\alpha} \phi\right] \rightarrow \mathcal{R}[\phi]$ such that

$$
\rho_{\alpha}\left([\beta]_{\tau_{\alpha} \phi}\right)=[\beta \alpha]_{\phi} .
$$

Linearizing (and again using the notation $\rho_{\alpha}$ ) gives a one-to-one correspondence

Thus we have

$$
\rho_{\alpha}: \mathbb{Z} \mathcal{R}\left[\tau_{\alpha} \phi\right] \longleftrightarrow \mathbb{Z} \mathcal{R}[\phi]
$$

$$
\begin{aligned}
\rho_{\alpha}(R T(f, \alpha \tilde{f})) & =\rho_{\alpha}\left(\sum_{[\beta]_{\tau_{\alpha} \phi} \in \mathcal{R}\left[\tau_{\alpha} \phi\right]} \operatorname{ind}\left(f ; \mathbb{F}_{[\beta]_{\tau_{\alpha} \phi}}\right)[\beta]_{\tau_{\alpha} \phi}\right) \\
& =\sum_{[\beta \alpha]_{\phi} \in \mathcal{R}[\phi]} \operatorname{ind}\left(f ; \mathbb{F}_{[\beta \alpha]_{\phi}}\right)[\beta \alpha]_{\phi} \\
& =\sum_{[\alpha]_{\phi} \in \mathcal{R}[\phi]} \operatorname{ind}\left(f ; \mathbb{F}_{[\alpha]_{\phi}}\right)[\alpha]_{\phi}
\end{aligned}
$$

and so $R T(f, \tilde{f})=\rho_{\alpha}(R T(f, \alpha \tilde{f}))$. That is, $R T(f, \tilde{f})$ and $R T(f, \alpha \tilde{f})$ are the same, except all Reidmeister representatives are multiplied on the right by $\alpha$.

By the same reason, we have

$$
R T(\bar{\alpha} \bar{f}, \alpha \tilde{f})=\rho_{\gamma}(R T(\bar{\alpha} \bar{f}, \gamma \alpha \tilde{f})), \quad \forall \gamma \in \Gamma .
$$

The following averaging formula for Reidemeister traces is the main result of [9]. Because the sum of the coefficients in the Reidiemeister trace equals the Lefschetz number, this is a direct generalization of Jiang's averaging formula for $L(f)$. We will give another proof using arguments from [7].

Theorem 1.3 ([9, Theorem 4.1]). For a mapping $f: X \rightarrow X$, choose some specific lift $\bar{f}: \bar{X} \rightarrow \bar{X}$. Then we have

$$
R T(f, \tilde{f})=\frac{1}{[\Pi: \Gamma]} \sum_{\bar{\alpha} \in \Pi / \Gamma} \rho_{\alpha} \circ \hat{i}^{\alpha}(R T(\bar{\alpha} \bar{f}, \alpha \tilde{f})) .
$$


Proof. Our proof follows the results and methods from [7]. For brevity in the following, we will omit subscripts on Reidemeister classes when they are obvious from context. For example $[\gamma]_{\tau_{\beta} \phi} \in \mathcal{R}\left[\tau_{\beta} \phi\right]$ will simply be written $[\gamma] \in \mathcal{R}\left[\tau_{\beta} \phi\right]$.

Recall that

$$
R T(f, \tilde{f})=\sum_{[\alpha] \in \mathcal{R}[\phi]} \operatorname{ind}\left(f ; \mathbb{F}_{[\alpha]}\right)[\alpha]=\sum_{[\alpha] \in \mathcal{R}[\phi]} \operatorname{ind}\left(f ; p(\operatorname{Fix}(\alpha \tilde{f}))[\alpha]_{\phi}\right.
$$

by the definition of $R T$ and $\mathbb{F}_{[\alpha]}$. Relabeling classes as in Lemma 1.1, for each $[\bar{\beta}] \in \mathcal{R}[\bar{\phi}]$ we fix a preimage $[\beta] \in \mathcal{R}[\phi]$, and the above becomes:

$$
R T(f, \tilde{f})=\sum_{[\bar{\beta}] \in \mathcal{R}[\bar{\phi}]}\left(\sum_{[\gamma] \in \operatorname{im}\left(\hat{i}^{\beta}\right)} \operatorname{ind}\left(f ; p(\operatorname{Fix}(\gamma \beta \tilde{f}))[\gamma \beta]_{\phi}\right) .\right.
$$

Rather than the inner sum over elements $[\gamma]$ of $\operatorname{im}\left(\hat{i}^{\beta}\right)$, we can sum over elements $\left[\gamma^{\prime}\right]$ of $\mathcal{R}\left[\tau_{\beta} \phi^{\prime}\right]$ with $\hat{i}^{\beta}\left(\left[\gamma^{\prime}\right]\right)=[\gamma]$. Since a single $\gamma \in \operatorname{im}\left(\hat{i}^{\beta}\right)$ may come from several elements in $\mathcal{R}\left[\tau_{\beta} \phi^{\prime}\right]$, we must insert the fraction below. Letting $F_{\gamma \beta}=p(\operatorname{Fix}(\gamma \beta \tilde{f}))$, we obtain:

$$
R T(f, \tilde{f})=\sum_{[\bar{\beta}] \in \mathcal{R}[\bar{\phi}]}\left(\sum_{\left[\gamma^{\prime}\right] \in \mathcal{R}\left[\tau_{\beta} \phi^{\prime}\right]} \frac{1}{\#\left(\hat{i}^{\beta}\right)^{-1}([\gamma])} \operatorname{ind}\left(f ; F_{\beta \gamma}\right)[\gamma \beta]_{\phi}\right) .
$$

By [7, Lemma 2.1], we also have

$$
\#\left(\hat{i}^{\beta}\right)^{-1}([\gamma])=\left[\operatorname{fix}\left(\tau_{\bar{\beta}} \bar{\phi}\right): u_{\gamma \beta}\left(\operatorname{fix}\left(\tau_{\gamma \beta} \phi\right)\right)\right],
$$

and thus we can rewrite the above as

$$
R T(f, \tilde{f})=\sum_{[\bar{\beta}] \in \mathcal{R}[\bar{\phi}]}\left(\sum_{\left[\gamma^{\prime}\right] \in \mathcal{R}\left[\tau_{\beta} \phi^{\prime}\right]} \frac{1}{\left[\operatorname{fix}\left(\tau_{\bar{\beta}} \bar{\phi}\right): u_{\gamma \beta}\left(\operatorname{fix}\left(\tau_{\gamma \beta} \phi\right)\right)\right]} \operatorname{ind}\left(f ; F_{\beta \gamma}\right)[\gamma \beta]_{\phi}\right) \text {. }
$$

Furthermore, we have

$$
\begin{aligned}
R T(f, \tilde{f}) & =\sum_{\bar{\beta} \in \Pi / \Gamma} \frac{1}{\#[\bar{\beta}]}\left(\sum_{\left[\gamma^{\prime}\right] \in \mathcal{R}\left[\tau_{\beta} \phi^{\prime}\right]} \frac{1}{\left[\operatorname{fix}\left(\tau_{\bar{\beta}} \bar{\phi}\right): u_{\gamma \beta}\left(\operatorname{fix}\left(\tau_{\gamma \beta} \phi\right)\right)\right]} \operatorname{ind}\left(f ; F_{\beta \gamma}\right)[\gamma \beta]_{\phi}\right) \\
& =\sum_{\bar{\beta} \in \Pi / \Gamma}\left(\sum_{\left[\gamma^{\prime}\right] \in \mathcal{R}\left[\tau_{\beta} \phi^{\prime}\right]} \frac{\# u_{\gamma \beta}\left(\operatorname{fix}\left(\tau_{\gamma \beta} \phi\right)\right)}{\#[\bar{\beta}] \cdot \# \operatorname{fix}\left(\tau_{\bar{\beta}} \bar{\phi}\right)} \operatorname{ind}\left(f ; F_{\beta \gamma}\right)[\gamma \beta]_{\phi}\right) .
\end{aligned}
$$

By [7, Remark 2.3], we have

$$
[\Pi: \Gamma]=\#[\bar{\beta}] \cdot \# \operatorname{fix}\left(\tau_{\bar{\beta}} \bar{\phi}\right), \forall \beta \in \Pi .
$$

Hence

$$
R T(f, \tilde{f})=\frac{1}{[\Pi: \Gamma]} \sum_{\bar{\beta} \in \Pi / \Gamma}\left(\sum_{\left[\gamma^{\prime}\right] \in \mathcal{R}\left[\tau_{\beta} \phi^{\prime}\right]} \# u_{\gamma \beta}\left(\operatorname{fix}\left(\tau_{\gamma \beta} \phi\right)\right) \operatorname{ind}\left(f ; F_{\beta \gamma}\right)[\gamma \beta]_{\phi}\right) .
$$


Now we remark that the fixed point class $p^{\prime}(\operatorname{Fix}(\gamma \beta \tilde{f}))$ of $\bar{\beta} \bar{f}$ covers by $\bar{p}$ the fixed point class $p(\operatorname{Fix}(\gamma \beta \tilde{f}))$ of $f$. By [7, Lemma 2.5], we see that each fixed point of $p(\operatorname{Fix}(\gamma \beta \tilde{f}))$ is covered by $\# u_{\gamma \beta}\left(\operatorname{fix}\left(\tau_{\gamma \beta} \phi\right)\right)$ fixed points of $p(\operatorname{Fix}(\gamma \beta \tilde{f}))$. Since the projections $p^{\prime}$ and $p$ are local homeomorphisms and the index is a local invariant, we have

$$
\operatorname{ind}\left(\beta \bar{f} ; p^{\prime}(\operatorname{Fix}(\gamma \beta \tilde{f}))\right)=\# u_{\gamma \beta}\left(\operatorname{fix}\left(\tau_{\gamma \beta} \phi\right)\right) \cdot \operatorname{ind}\left(f ; F_{\beta \gamma}\right),
$$

and consequently, we obtain

$$
R T(f, \tilde{f})=\frac{1}{[\Pi: \Gamma]} \sum_{\bar{\beta} \in \Pi / \Gamma}\left(\sum_{\left[\gamma^{\prime}\right] \in \mathcal{R}\left[\tau_{\beta} \phi^{\prime}\right]} \operatorname{ind}\left(\bar{\beta} \bar{f} ; p^{\prime}(\operatorname{Fix}(\gamma \beta \tilde{f}))[\gamma \beta]_{\phi}\right) .\right.
$$

We know that $[\gamma \beta]_{\phi}=\rho_{\beta}\left([\gamma]_{\tau_{\beta} \phi}\right)$, and by the definition of $\gamma^{\prime}$ we have $[\gamma]_{\tau_{\beta} \phi}=\hat{i}^{\beta}\left(\left[\gamma^{\prime}\right]_{\tau_{\beta} \phi^{\prime}}\right)$, and thus the above gives:

$$
\begin{aligned}
R T(f, \tilde{f}) & =\frac{1}{[\Pi: \Gamma]} \sum_{\bar{\beta} \in \Pi / \Gamma}\left(\sum_{\left[\gamma^{\prime}\right] \in \mathcal{R}\left[\tau_{\beta} \phi^{\prime}\right]} \operatorname{ind}\left(\bar{\beta} \bar{f} ; p^{\prime}(\operatorname{Fix}(\gamma \beta \tilde{f}))\right) \rho_{\beta}\left(\hat{i}^{\beta}\left(\left[\gamma^{\prime}\right]_{\tau_{\beta} \phi^{\prime}}\right)\right)\right) \\
& =\frac{1}{[\Pi: \Gamma]} \sum_{\bar{\beta} \in \Pi / \Gamma} \rho_{\beta} \circ \hat{i}^{\beta}\left(\sum_{\left[\gamma^{\prime}\right] \in \mathcal{R}\left[\tau_{\beta} \phi^{\prime}\right]} \operatorname{ind}\left(\bar{\beta} \bar{f} ; p^{\prime}(\operatorname{Fix}(\gamma \beta \tilde{f}))\left[\gamma^{\prime}\right]_{\tau_{\beta} \phi^{\prime}}\right)\right. \\
& =\frac{1}{[\Pi: \Gamma]} \sum_{\bar{\beta} \in \Pi / \Gamma} \rho_{\beta} \circ \hat{i}^{\beta}(R T(\bar{\beta} \bar{f}, \beta \tilde{f})) .
\end{aligned}
$$

\section{Coincidence theory}

Let $M_{1}$ and $M_{2}$ be closed orientable manifolds of equal dimension $n$. We will generalize the general setup of the previous section to the setting of pairs of mappings $f, g: M_{1} \rightarrow M_{2}$ and the coincidence set

$$
\operatorname{Coin}(f, g)=\left\{x \in M_{1} \mid f(x)=g(x)\right\} .
$$

Let $p_{1}: \tilde{M}_{1} \rightarrow M_{1}$ and $p_{2}: \tilde{M}_{2} \rightarrow M_{2}$ be the universal covering projections with the groups of covering transformations $\Pi_{1}$ and $\Pi_{2}$ respectively. By fixing lifts $\tilde{f}$ and $\tilde{g}$ of $f$ and $g$, the continuous maps $f$ and $g$ induce homomorphisms $\phi: \Pi_{1} \rightarrow \Pi_{2}$ and $\psi: \Pi_{1} \rightarrow \Pi_{2}$ between the groups of covering transformations as follows.

$$
\phi(\alpha) \tilde{f}=\tilde{f} \alpha, \psi(\alpha) \tilde{g}=\tilde{g} \alpha, \quad \forall \alpha \in \Pi_{1} .
$$

The set of Reidemeister classes $\mathcal{R}[\phi, \psi]$ is defined as the quotient of $\Pi_{2}$ by the doubly-twisted conjugacy relation $\alpha \sim \beta$ if and only if there is some $\gamma \in \Pi_{1}$ with $\alpha=\psi(\gamma)^{-1} \beta \phi(\gamma)$. The Reidemeister class containing $\beta$ will be written as $[\beta] \in \mathcal{R}[\phi, \psi]$, or when we wish to emphasize the homomorphisms, as $[\beta]_{\phi, \psi}$. 
Then it is well known that the coincidence set $\operatorname{Coin}(f, g)$ splits into a disjoint union of coincidence classes. That is,

$$
\operatorname{Coin}(f, g)=\bigsqcup_{[\beta] \in \mathcal{R}[\phi, \psi]} p_{1}(\operatorname{Coin}(\beta \tilde{f}, \tilde{g})) .
$$

Thus any coincidence class $\mathbb{S}=p_{1}(\operatorname{Coin}(\beta \tilde{f}, \tilde{g}))$ can be indexed by the Reidemeister class $[\beta] ; \mathbb{S}=\mathbb{S}_{[\beta]}$.

Definition 2.1. The coincidence Reidemeister trace of $(f, g)$ is defined as follows:

$$
\begin{aligned}
R T(f, \tilde{f}, g, \tilde{g}) & =\sum_{[\beta] \in \mathcal{R}[\phi, \psi]} \operatorname{ind}\left(f, g ; \mathbb{S}_{[\beta]}\right)[\beta] \\
& =\sum_{[\beta] \in \mathcal{R}[\phi, \psi]} \operatorname{ind}\left(f, g ; p_{1}(\operatorname{Coin}(\beta \tilde{f}, \tilde{g}))\right)[\beta] \in \mathbb{Z} \mathcal{R}[\phi, \psi] .
\end{aligned}
$$

where ind denotes the coincidence index of a coincidence class.

We remark that the number of non-zero terms in this sum is the Nielsen coincidence number $N(f, g)$, and the sum of the coefficients is the Lefschetz coincidence number $L(f, g)$.

For $\beta \in \Pi_{2}, \beta \tilde{f}$ is another lift of $f$. Then the homomorphism induced by $f$ by using the lift $\beta \tilde{f}$ is $\tau_{\beta} \phi: \Pi_{1} \rightarrow \Pi_{2}$, where $\tau_{\beta}$ denotes conjugation by $\beta$.

As in fixed point theory, the quantities $R T(f, \tilde{f}, g, \tilde{g})$ and $R T(f, \beta \tilde{f}, g, \tilde{g})$ are related by the map $\rho_{\beta}: \mathcal{R}\left[\tau_{\beta} \phi, \psi\right] \rightarrow \mathcal{R}[\phi, \psi]$ defined by

$$
\rho_{\beta}\left([\gamma]_{\tau_{\beta} \phi, \psi}\right)=[\gamma \beta]_{\phi, \psi} .
$$

It can be verified as in fixed point theory that:

$$
R T(f, \tilde{f}, g, \tilde{g})=\rho_{\beta}(R T(f, \beta \tilde{f}, g, \tilde{g})) .
$$

Let $\Gamma_{1}$ and $\Gamma_{2}$ be finite index normal subgroups of $\Pi_{1}$ and $\Pi_{2}$ so that $\phi\left(\Gamma_{1}\right) \subset \Gamma_{2}$ and $\psi\left(\Gamma_{1}\right) \subset \Gamma_{2}$. For each $\bar{\beta} \in \Pi_{1} / \Gamma_{2}$ and $\beta \in u_{2}^{-1}(\bar{\beta})$, we have the following commutative diagram

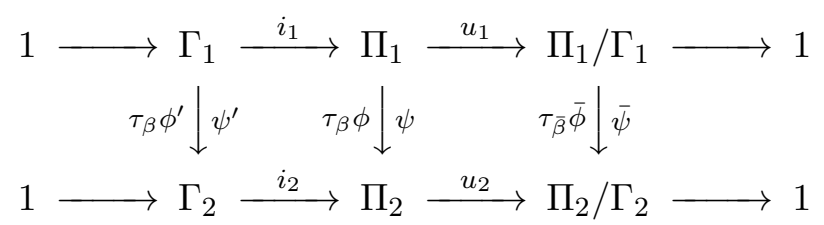

Moreover the following sequence of groups

$$
1 \rightarrow \operatorname{coin}\left(\tau_{\beta} \phi^{\prime}, \psi^{\prime}\right) \stackrel{i_{1}^{\beta}}{\longrightarrow} \operatorname{coin}\left(\tau_{\beta} \phi, \psi\right) \stackrel{u_{1}^{\beta}}{\longrightarrow} \operatorname{coin}\left(\tau_{\bar{\beta}} \bar{\phi}, \bar{\psi}\right)
$$

is exact, where coin denotes the coincidence subgroup of a pair of homomorphisms. We remark also that $i_{2}: \Gamma_{2} \rightarrow \Pi_{2}$ and $u_{2}: \Pi_{2} \rightarrow \Pi_{2} / \Gamma_{2}$ induce maps between the Reidemeister sets $\hat{i}_{2}^{\beta}: \mathcal{R}\left[\tau_{\beta} \phi^{\prime}, \psi^{\prime}\right] \rightarrow \mathcal{R}\left[\tau_{\beta} \phi, \psi\right]$ and $\hat{u}_{2}^{\beta}$ : 
$\mathcal{R}\left[\tau_{\beta} \phi, \psi\right] \rightarrow \mathcal{R}\left[\tau_{\bar{\beta}} \bar{\phi}, \bar{\psi}\right]$ such that $\hat{u}_{2}^{\beta}$ is surjective and $\left(\hat{u}_{2}^{\beta}\right)^{-1}([\overline{1}])=\operatorname{im}\left(\hat{i}_{2}^{\beta}\right)$. That is, the following sequence of sets is exact:

$$
\mathcal{R}\left[\tau_{\beta} \phi^{\prime}, \psi^{\prime}\right] \stackrel{\hat{i}_{2}^{\beta}}{\longrightarrow} \mathcal{R}\left[\tau_{\beta} \phi, \psi\right] \stackrel{\hat{u}_{2}^{\beta}}{\longrightarrow} \mathcal{R}\left[\tau_{\bar{\beta}} \bar{\phi}, \bar{\psi}\right] \longrightarrow 1 .
$$

Denoting $\bar{M}_{1}=\Gamma_{1} \backslash \tilde{M}_{1}$ and $\bar{M}_{2}=\Gamma_{2} \backslash \tilde{M}_{2}$, we see that $\bar{M}_{1}$ and $\bar{M}_{2}$ are regular coverings of $M_{1}$ and $M_{2}$ such that

$$
\begin{aligned}
& p_{1}: \tilde{M}_{1} \stackrel{p_{1}^{\prime}}{\longrightarrow} \bar{M}_{1} \stackrel{\bar{p}_{1}}{\longrightarrow} M_{1}, \\
& p_{2}: \tilde{M}_{2} \stackrel{p_{2}^{\prime}}{\longrightarrow} \bar{M}_{2} \stackrel{\bar{p}_{2}}{\longrightarrow} M_{2}
\end{aligned}
$$

are covering projections.

Since $\phi\left(\Gamma_{1}\right) \subset \Gamma_{2}$ and $\psi\left(\Gamma_{1}\right) \subset \Gamma_{2}$, the pair of lifts $(\tilde{f}, \tilde{g})$ induces the pair of lifts $(\bar{f}, \bar{g})$ of $(f, g)$ so that for any $\bar{\beta} \in \Pi_{1} / \Gamma_{2}$ and $\beta \in u_{2}^{-1}(\bar{\beta})$, the following diagram is commutative

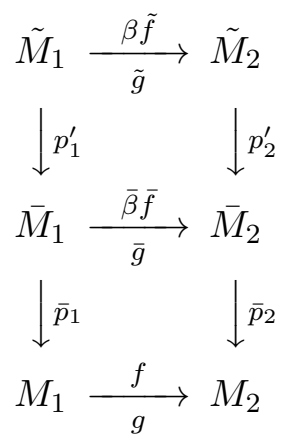

We remark also that the pair of homomorphisms $\left(\tau_{\beta} \phi^{\prime}, \psi^{\prime}\right)$ are the homomorphisms induced by $(\bar{\beta} \bar{f}, \bar{g})$ by fixing the lifts $(\beta \tilde{f}, \tilde{g})$. Similarly, the pair of homomorphisms $\left(\tau_{\bar{\beta}} \bar{\phi}, \bar{\psi}\right)$ are the homomorphisms induced by $(f, g)$ by fixing the lifts $(\bar{\beta} \bar{f}, \bar{g})$.

Therefore, the coincidence Reidemeister trace of $(\bar{\beta} \bar{f}, \bar{g})$ is by definition

$$
\begin{aligned}
& R T(\bar{\beta} \bar{f}, \beta \tilde{f}, \bar{g}, \tilde{g}) \\
& =\sum_{[\gamma] \in \mathcal{R}\left[\tau_{\beta} \phi^{\prime}, \psi^{\prime}\right]} \operatorname{ind}\left(\bar{\beta} \bar{f}, \bar{g} ; p_{1}^{\prime}(\operatorname{Coin}(\gamma(\beta \tilde{f}), \tilde{g}))[\gamma] \in \mathbb{Z} \mathcal{R}\left[\tau_{\beta} \phi^{\prime}, \psi^{\prime}\right] .\right.
\end{aligned}
$$

As in fixed point theory, there is an averaging formula for the Lefschetz coincidence number. The following formula is apparently due to McCord:

$$
L(f, g)=\frac{1}{\left[\Pi_{1}: \Gamma_{1}\right]} \sum_{\bar{\beta} \in \Pi_{2} / \Gamma_{2}} L(\bar{\beta} \bar{f}, \bar{g}) .
$$

The summation on the right side above is discussed in [10, page 360], and the full formula appears in [11, page 88]. The corresponding averaging formula for Nielsen numbers does not hold in general, this is studied in [8]. 


\section{MAin RESUlt}

The following averaging formula for the coincidence Reidemeister trace is our main result:

Theorem 3.1. For maps $f, g: M_{1} \rightarrow M_{2}$, choose some specific lifts $\bar{f}, \bar{g}:$ $\bar{M}_{1} \rightarrow \bar{M}_{2}$. We have

$$
R T(f, \tilde{f}, g, \tilde{g})=\frac{1}{\left[\Pi_{1}: \Gamma_{1}\right]} \sum_{\bar{\beta} \in \Pi_{2} / \Gamma_{2}} \rho_{\beta} \circ \hat{i}_{2}^{\beta}(R T(\bar{\beta} \bar{f}, \beta \tilde{f}, \bar{g}, \tilde{g})) .
$$

Proof. The proof follows exactly the structure of the proof of Theorem 1.3. substituting the required results in coincidence theory from 8 .

Lemma 2.2 of [8] gives a coincidence version of Lemma 1.1. Specifically the coincidence classes decompose as follows:

$$
\operatorname{Coin}(f, g)=\bigsqcup_{[\bar{\beta}] \in \mathcal{R}[\bar{\phi}, \bar{\psi}]} \bigsqcup_{[\gamma]_{\tau_{\beta}, \psi} \in \operatorname{im}\left(\hat{i}_{2}^{\beta}\right)} p_{1}(\operatorname{Coin}(\gamma \beta \tilde{f}, \tilde{g})) .
$$

Letting $S_{\gamma \beta}=p_{1}(\operatorname{Coin}(\gamma \beta \tilde{f}, \tilde{g}))$, we may relabel coincidence classes and we have:

$$
R T(f, \tilde{f}, g, \tilde{g})=\sum_{[\bar{\beta}] \in \mathcal{R}[\bar{\phi}, \bar{\psi}]}\left(\sum_{[\gamma]_{\tau_{\beta} \phi, \psi} \in \operatorname{im}\left(\hat{i}_{2}^{\beta}\right)} \operatorname{ind}\left(f, g ; S_{\gamma \beta}\right)[\gamma \beta]_{\phi, \psi}\right) .
$$

As in the proof of Theorem 1.3 we obtain:

$$
R T(f, \tilde{f}, g, \tilde{g})=\sum_{[\bar{\beta}] \in \mathcal{R}[\bar{\phi}, \bar{\psi}]}\left(\sum_{\left[\gamma^{\prime}\right] \in \mathcal{R}\left[\tau_{\beta} \phi^{\prime}, \psi^{\prime}\right]} \frac{1}{\#\left(\hat{i}_{2}^{\beta}\right)^{-1}([\gamma])} \operatorname{ind}\left(f, g ; S_{\gamma \beta}\right)[\gamma \beta]_{\phi, \psi}\right) \text {. }
$$

By [8, Lemma 2.1], we also have

$$
\#\left(\hat{i}_{2}^{\beta}\right)^{-1}([\gamma])=\left[\operatorname{coin}\left(\tau_{\bar{\beta}} \bar{\phi}, \bar{\psi}\right): u_{\gamma \beta}\left(\operatorname{coin}\left(\tau_{\gamma \beta} \phi, \psi\right)\right)\right]=\frac{\# \operatorname{coin}\left(\tau_{\bar{\beta}} \bar{\phi}, \bar{\psi}\right)}{\# u_{\gamma \beta}\left(\operatorname{coin}\left(\tau_{\gamma \beta} \phi, \psi\right)\right)}
$$

and thus we can rewrite the above as

$$
\begin{aligned}
R T(f, \tilde{f}, g, \tilde{g}) & =\sum_{[\bar{\beta}] \in \mathcal{R}[\bar{\phi}, \bar{\psi}]}\left(\sum_{\left[\gamma^{\prime}\right] \in \mathcal{R}\left[\tau_{\beta} \phi^{\prime}, \psi^{\prime}\right]} \frac{\# u_{\gamma \beta}\left(\operatorname{coin}\left(\tau_{\gamma \beta} \phi, \psi\right)\right)}{\# \operatorname{coin}\left(\tau_{\bar{\beta}} \bar{\phi}, \bar{\psi}\right)} \operatorname{ind}\left(f, g ; S_{\gamma \beta}\right)[\gamma \beta]_{\phi, \psi}\right) \\
& =\sum_{\bar{\beta} \in \Pi_{2} / K}\left(\sum_{\left[\gamma^{\prime}\right] \in \mathcal{R}\left[\tau_{\beta} \phi^{\prime}, \psi^{\prime}\right]} \frac{\# u_{\gamma \beta}\left(\operatorname{coin}\left(\tau_{\gamma \beta} \phi, \psi\right)\right)}{\#[\bar{\beta}] \cdot \# \operatorname{coin}\left(\tau_{\bar{\beta}} \bar{\phi}, \bar{\psi}\right)} \operatorname{ind}\left(f, g ; S_{\gamma \beta}\right)[\gamma \beta]_{\phi, \psi}\right) .
\end{aligned}
$$

On each Reidemeister class $[\bar{\beta}]$ of $\mathcal{R}[\bar{\phi}, \bar{\psi}]$, the group $\Pi_{1} / \Gamma_{1}$ acts transitively by the rule $\bar{\beta} \mapsto \bar{\psi}(\bar{\delta}) \bar{\beta} \bar{\phi}(\bar{\delta})^{-1}$. The isotropy subgroup at $\bar{\beta}$ is

$$
\left\{\bar{\delta} \mid \bar{\psi}(\bar{\delta}) \bar{\beta} \bar{\phi}(\bar{\delta})^{-1}=\bar{\beta}\right\}=\operatorname{coin}\left(\tau_{\bar{\beta}} \bar{\phi}, \bar{\psi}\right) .
$$

Thus

$$
\left[\Pi_{1}: \Gamma_{1}\right]=\#[\bar{\beta}] \cdot \# \operatorname{coin}\left(\tau_{\bar{\beta}} \bar{\phi}, \bar{\psi}\right), \forall[\bar{\beta}] \in \mathcal{R}[\bar{\phi}, \bar{\psi}]
$$


and so

$R T(f, \tilde{f}, g, \tilde{g})=\frac{1}{\left[\Pi_{1}: \Gamma_{1}\right]} \sum_{\bar{\beta} \in \Pi_{2} / K}\left(\sum_{\left[\gamma^{\prime}\right] \in \mathcal{R}\left[\tau_{\beta} \phi^{\prime}, \psi^{\prime}\right]} \# u_{\gamma \beta}\left(\operatorname{coin}\left(\tau_{\gamma \beta} \phi, \psi\right)\right) \operatorname{ind}\left(f, g ; S_{\gamma \beta}\right)[\gamma \beta]_{\phi, \psi}\right)$.

Now we remark that the coincidence class $p_{1}^{\prime}(\operatorname{Coin}(\gamma \beta \tilde{f}, \tilde{g}))$ of $(\bar{\beta} \bar{f}, \bar{g})$ covers by $\bar{p}_{1}$ the coincidence class $S_{\gamma \beta}=p_{1}(\operatorname{Coin}(\gamma \beta \tilde{f}, \tilde{g}))$ of $(f, g)$. By Lemma 4.2 of [8] we see that this cover is $\# u_{1}\left(\operatorname{coin}\left(\tau_{\gamma \beta} \phi, \psi\right)\right)$-fold. Since the projections $p_{1}^{\prime}$ and $p_{1}$ are orientation preserving local homeomorphisms and the index is a local invariant, we have

$$
\operatorname{ind}\left(\beta \bar{f}, \bar{g} ; p_{1}^{\prime}(\operatorname{Coin}(\gamma \beta \tilde{f}, \tilde{g}))\right)=\# u_{\gamma \beta}\left(\operatorname{coin}\left(\tau_{\gamma \beta} \phi, \psi\right)\right) \cdot \operatorname{ind}\left(f, g ; S_{\gamma \beta}\right),
$$

Therefore, the above identity reduces to:

$$
\begin{aligned}
R T(f, \tilde{f}, g, \tilde{g}) & =\frac{1}{\left[\Pi_{1}: \Gamma_{1}\right]} \sum_{\bar{\beta} \in \Pi_{2} / K}\left(\sum_{\left[\gamma^{\prime}\right] \in \mathcal{R}\left[\tau_{\beta} \phi^{\prime}, \psi^{\prime}\right]} \operatorname{ind}\left(\bar{\beta} \bar{f}, \bar{g} ; S_{\gamma \beta}\right)[\gamma \beta]_{\phi, \psi}\right) \\
& =\frac{1}{\left[\Pi_{1}: \Gamma_{1}\right]} \sum_{\bar{\beta} \in \Pi_{2} / K} \rho_{\beta} \circ \hat{i}_{2}^{\beta}\left(\sum_{\left[\gamma^{\prime}\right] \in \mathcal{R}\left[\tau_{\beta} \phi^{\prime}, \psi^{\prime}\right]} \operatorname{ind}\left(\bar{\beta} \bar{f}, \bar{g} ; S_{\gamma \beta}\right)\left[\gamma^{\prime}\right]_{\phi^{\prime}, \psi^{\prime}}\right) \\
& =\frac{1}{\left[\Pi_{1}: \Gamma_{1}\right]} \sum_{\bar{\beta} \in \Pi_{2} / K} \rho_{\beta} \circ \hat{i}_{2}^{\beta}(R T(\bar{\beta} \bar{f}, \beta \tilde{f}, \bar{g}, \tilde{g})) .
\end{aligned}
$$

\section{An AXIOMATIC PROOF}

The averaging formula for the coincidence Reidemeister trace can also be obtained as an application of a uniqueness theorem from [13]. This uniqueness result was a direct generalization of earlier work in [2, 12, 13] concerning the local fixed point and coincidence index. To demonstrate the basic idea, we begin with an axiomatic proof of an averaging formula for the local fixed point index.

Recall that the local fixed point index $\operatorname{ind}(f ; U)$ is an integer invariant which is defined for any open set $U$ such that $\operatorname{Fix}(f) \cap U$ is compact.

Let $M$ be a compact differentiable manifold with universal covering $p$ : $\tilde{M} \rightarrow M$ and a finite covering $\bar{p}: \bar{M} \rightarrow M$ with the natural map $p^{\prime}: \tilde{M} \rightarrow \bar{M}$ with $p=p^{\prime} \circ \bar{p}$.

When $U \subset M$ is an open set, and $f: U \rightarrow M$ is a continuous map, say $(f ; U)$ is admissible when $\operatorname{Fix}(f) \cap U$ is compact, and let $\mathcal{C}(M)$ be the set of all admissible pairs on $M$. If $H: M \times[0,1] \rightarrow M$ is a homotopy, we say that $H$ is admissible in $U$ if the set

$$
\{(x, t) \mid H(x, t)=x\}
$$

is compact in $M \times[0,1]$.

The following is a result of Furi, Pera, and Spadini [2]. 
Theorem 4.1. Let $M$ be a compact differentiable manifold, and let $\iota$ : $\mathcal{C}(M) \rightarrow \mathbb{R}$ be any function satisfying the following 3 axioms:

- (Homotopy) If there is a homotopy from $(f ; U)$ to $\left(f^{\prime} ; U\right)$ which is admissible in $U$, then $\iota(f ; U)=\iota\left(f^{\prime} ; U\right)$.

- (Additivity) If $U_{1}, U_{2}$ are disjoint open subsets of $U$ with $\operatorname{Fix}(f) \cap U \subset$ $U_{1} \sqcup U_{2}$, then

$$
\iota\left(f ; U_{1}\right)+\iota\left(f ; U_{2}\right)=\iota(f ; U) .
$$

- (Normalization) If $c$ is a constant map, then $\iota(c ; M)=1$

Then $\iota$ is the local fixed point index.

We will use the uniqueness theorem above to obtain the following averaging formula for the local fixed point index.

Theorem 4.2. Let ind $(f ; U)$ be the local fixed point index of an admissible pair, and write $\bar{U}=p^{-1}(U)$. For any specific lift $\bar{f}: \bar{M} \rightarrow \bar{M}$ of $f$, we have

$$
\operatorname{ind}(f ; U)=\frac{1}{[\Pi: \Gamma]} \sum_{\bar{\beta} \in \Pi / \Gamma} \operatorname{ind}(\bar{\beta} \bar{f} ; \bar{U}) .
$$

Proof. Before proving formula (4-1) we should note that when $\operatorname{Fix}(f) \cap U$ is compact in $M$, since $q$ is a covering map we will have $\operatorname{Fix}(\bar{\beta} \bar{f}) \cap \bar{U}$ compact in $\bar{M}$ for any lift $\bar{f}$ of $f$, and thus the pair $(\bar{f}, \bar{U})$ is admissible and so the index on the right side of (4-1) is defined.

For any admissible pair $(f ; U)$, let

$$
\iota(f ; U)=\frac{1}{[\Pi: \Gamma]} \sum_{\bar{\beta} \in \Pi / \Gamma} \operatorname{ind}(\bar{\beta} \bar{f} ; \bar{U}),
$$

where $\bar{f}$ is any lift of $f$. It suffices to show that $\iota$ satisfies the three axioms of Theorem 4.1.

First we remark that $\iota$ is well defined (independent of the choice of lift $\bar{f})$. It suffices to show that if $\bar{f}$ and $\hat{f}$ are two different choices of lifts, then

$$
\sum_{\bar{\beta} \in \Pi / \Gamma} \operatorname{ind}(\bar{\beta} \bar{f} ; \bar{U})=\sum_{\bar{\beta} \in \Pi / \Gamma} \operatorname{ind}(\bar{\beta} \hat{f} ; \bar{U}) .
$$

There is some $\bar{\alpha} \in \Pi / \Gamma$ with $\bar{\alpha} \hat{f}=\bar{f}$, and so we have:

$$
\sum_{\bar{\beta} \in \Pi / \Gamma} \operatorname{ind}(\bar{\beta} \bar{f} ; \bar{U})=\frac{1}{[\Pi: \Gamma]} \sum_{\bar{\beta} \in \Pi / \Gamma} \operatorname{ind}(\bar{\beta} \bar{\alpha} \hat{f} ; \bar{U})=\frac{1}{[\Pi: \Gamma]} \sum_{\bar{\beta} \in \Pi / \Gamma} \operatorname{ind}(\bar{\beta} \hat{f} ; \bar{U})
$$

as desired. Now we show that $\iota$ satisfies the three axioms of Theorem 4.1 ,

For the homotopy axiom, let $H$ be an admissible homotopy from $(f ; U)$ to $\left(f^{\prime} ; U\right)$. Then for any lift $\bar{\beta} \bar{f}$ of $f$, the homotopy $H$ will lift to a unique homotopy $\bar{H}$ from $\bar{\beta} \bar{f}$ to some lift $\bar{\beta} \bar{f}^{\prime}$ of $f^{\prime}$, and it can be checked that this 
$\bar{H}$ is admissible in $\bar{U}$. Thus we have

$$
\iota(f ; U)=\frac{1}{[\Pi: \Gamma]} \sum_{\bar{\beta} \in \Pi / \Gamma} \operatorname{ind}(\bar{\beta} \bar{f} ; \bar{U})=\frac{1}{[\Pi: \Gamma]} \sum_{\bar{\beta} \in \Pi / \Gamma} \operatorname{ind}\left(\bar{\beta} \bar{f}^{\prime} ; \bar{U}\right)=\iota\left(f^{\prime} ; U\right)
$$

and so $\iota$ satisfies the homotopy axiom.

For the additivity axiom, let $(f ; U) \in \mathcal{C}(M)$ with $\operatorname{Fix}(f) \cap U \subset U_{1} \sqcup U_{2}$. Then for any lift $\bar{f}$ of $f$ we have:

$$
\operatorname{Fix}(\bar{f}) \cap \bar{U} \subset \bar{p}^{-1}(\operatorname{Fix}(f) \cap U) \subset \bar{p}^{-1}\left(U_{1} \sqcup U_{2}\right)=\bar{U}_{1} \sqcup \bar{U}_{2} .
$$

Thus the additivity axiom for $\operatorname{ind}(\bar{\beta} \bar{f} ; \bar{U})$ gives:

$$
\begin{aligned}
\iota(f ; U) & =\frac{1}{[\Pi: \Gamma]} \sum_{\bar{\beta} \in \Pi / \Gamma} \operatorname{ind}(\bar{\beta} \bar{f} ; \bar{U}) \\
& \left.=\frac{1}{[\Pi: \Gamma]} \sum_{\bar{\beta} \in \Pi / \Gamma} \operatorname{ind}\left(\bar{\beta} \bar{f} ; \bar{U}_{1}\right)+\operatorname{ind}\left(\bar{\beta} \bar{f} ; \bar{U}_{2}\right)\right) \\
& =\frac{1}{[\Pi: \Gamma]} \sum_{\bar{\beta} \in \Pi / \Gamma} \operatorname{ind}\left(\bar{\beta} \bar{f} ; \bar{U}_{1}\right)+\frac{1}{[\Pi: \Gamma]} \sum_{\bar{\beta} \in \Pi / \Gamma} \operatorname{ind}\left(\bar{\beta} \bar{f} ; \bar{U}_{2}\right) \\
& =\iota\left(f ; U_{1}\right)+\iota\left(f ; U_{2}\right),
\end{aligned}
$$

and thus $\iota$ satisfies the additivity axiom.

For the normalization axiom, let $c: M \rightarrow M$ be a constant map. Then any lift $\bar{c}: \bar{M} \rightarrow \bar{M}$ is a constant map on $\bar{M}$, and so $\bar{\beta} \bar{c}$ is a constant map for any $\bar{\beta}$. Thus using the normalization axiom on $\operatorname{ind}(\bar{\beta} \bar{c}, \bar{M})$ gives:

$$
\iota(c ; M)=\frac{1}{[\Pi: \Gamma]} \sum_{\bar{\beta} \in \Pi / \Gamma} \operatorname{ind}(\bar{\beta} \bar{c} ; \bar{M})=\frac{1}{[\Pi: \Gamma]} \sum_{\bar{\beta} \in \Pi / \Gamma} 1=1 .
$$

Thus $\iota$ satisfies the normalization axiom.

Since $\iota$ satisfies the three axioms, it must be the fixed point index.

If we take $U=M$ above we obtain a new proof of the classical Lefschetz averaging formula, since $\operatorname{ind}(f ; M)=L(f)$.

An averaging formula like the one in Theorem 4.2 can be obtained for the local coincidence index, by substituting the uniqueness theorem for the coincidence index from [12] in place of Theorem 4.1. The uniqueness theorems in [13] can be used to obtain similar averaging formulas for the local Reidmeister trace and local coincidence Reidemeister trace. We will prove only the most general of these, the local coincidence Reidemeister trace.

Theorem 4.1 was generalized to the coincidence Reidemeister trace in [13] as follows: Let $M_{1}$ and $M_{2}$ be oriented closed manifolds of the same dimension, let $\tilde{M}_{1}$ and $\tilde{M}_{2}$ be their universal covers, and let $\mathcal{C}\left(M_{1}, M_{2}\right)$ be the set of admissible tuples $(f, \tilde{f}, g, \tilde{g} ; U)$ where $f, g: M_{1} \rightarrow M_{2}$ are continuous, $\tilde{f}, \tilde{g}: \tilde{M}_{1} \rightarrow \tilde{M}_{2}$ are lifts of $f$ and $g$, and $U$ is an open set with $\operatorname{Coin}(f, g) \cap U$ 
compact. If $H, G: M_{1} \times[0,1] \rightarrow M_{2}$ are homotopies, we say the pair $(H, G)$ is admissible in $U$ when the set

$$
\left\{(x, t) \in M_{1} \times[0,1] \mid H(x, t)=G(x, t)\right\}
$$

is compact.

The local coincidence Reidemeister trace is defined as in Definition $2-2$, but restricting only to the subset $U$ :

$$
R T(f, \tilde{f}, g, \tilde{g} ; U)=\sum_{[\beta] \in \mathcal{R}[\phi, \psi]} \operatorname{ind}\left(\left.f\right|_{U},\left.g\right|_{U} ;\left.\mathbb{S}_{[\beta]}\right|_{U}\right)[\beta],
$$

where $\left.f\right|_{U}$ and $\left.g\right|_{U}$ denote the restrictions of $f$ and $g$ to $U$, and

$$
\left.\mathbb{S}_{[\beta]}\right|_{U}=p_{1}\left(\operatorname{Coin}\left(\left.\beta \tilde{f}\right|_{\tilde{U}},\left.\tilde{g}\right|_{\tilde{U}}\right)\right), \quad \tilde{U}=p_{1}^{-1}(U) .
$$

It is clear from the definition that $R T\left(f, \tilde{f}, g, \tilde{g} ; M_{1}\right)=R T(f, \tilde{f}, g, \tilde{g})$.

The manifolds in [2, 12, 13] are always assumed to be differentiable. This assumption is made in order to use transversality arguments, but is not necessary. Jezierski proves a "topological transversality lemma" in [5] which provides the same results for topological manifolds. The work in [3] generalizes all results from [2, 12, 13] without the differentiability assumption, so we will not require differentiability in this paper. We will also use the normalization axiom used in [3], which is weaker than the one from [13] based on the Lefschetz number. In our normalization property we assume that $M_{1}$ and $M_{2}$ have fixed chosen orientations.

When $S$ is any set, let $\epsilon: \mathbb{Z} S \rightarrow \mathbb{Z}$ be the augmentation map (sum of coefficients).

Theorem 4.3 ([13], Theorem 3; 3], Theorem 44). Let $\sigma$ be a function with domain $\mathcal{C}\left(M_{1}, M_{2}\right)$ such that $\sigma(f, \tilde{f}, g, \tilde{g} ; U) \in \mathbb{Z} \mathcal{R}[\phi, \psi]$, where $\phi$ and $\psi$ are the induced homomorphisms of $f$ and $g$ respectively. Assume that $\sigma$ satisfies the following axioms:

- (Homotopy) If there is a pair of homotopies from $f$ to $f^{\prime}$ and $g$ to $g^{\prime}$ which is admissible in $U$, then $\sigma(f, \tilde{f}, g, \tilde{g} ; U)=\sigma\left(f^{\prime}, \tilde{f}^{\prime}, g^{\prime}, \tilde{g}^{\prime} ; U\right)$, where $\tilde{f}^{\prime}$ and $\tilde{g}^{\prime}$ are the unique lifts of $f^{\prime}$ and $g^{\prime}$ obtained by lifting the homotopies to $\tilde{f}$ and $\tilde{g}$.

- (Additivity) If $U_{1}, U_{2}$ are disjoint open subsets of $U$ with $\operatorname{Coin}(f, g) \cap$ $U \subset U_{1} \sqcup U_{2}$, then

$$
\sigma(f, \tilde{f}, g, \tilde{g} ; U)=\sigma\left(f, \tilde{f}, g, \tilde{g} ; U_{1}\right)+\sigma\left(f, \tilde{f}, g, \tilde{g} ; U_{2}\right)
$$

- (Normalization) Let $c: M_{1} \rightarrow M_{2}$ be a constant map with constant value $c \in M_{2}$, and $g: U \rightarrow M_{2}$ be an orientation preserving embedding with $g(x)=c$. (That is, $g$ carries the positive orientation on $U$ to the positive orientation on $g(U)$.) Then:

$$
\epsilon(\sigma(c, \tilde{c}, g, \tilde{g} ; U))=1 .
$$


- (Lift invariance) For any $\alpha, \beta \in \Pi_{2}$, we have

$$
\epsilon(\sigma(f, \tilde{f}, g, \tilde{g} ; U))=\epsilon(\sigma(f, \alpha \tilde{f}, g, \beta \tilde{g} ; U))
$$

- (Coincidence of lifts) If $[\alpha]$ appears in $\sigma(f, \tilde{f}, g, \tilde{g} ; U)$ with nonzero coefficient, then $\alpha \tilde{f}$ and $\tilde{g}$ have a coincidence on $p_{1}^{-1}(U)$.

Then $\sigma$ is the local coincidence Reidemeister trace.

Our averaging formula for the local coincidence Reidemeister trace is proved similarly to Theorem 4.2 .

Theorem 4.4. For any $U \subset M_{1}$, let $\bar{U}=\bar{p}_{1}^{-1}(U) \subset \bar{M}_{1}$ and let $\bar{f}, \bar{g}$ be specific chosen lifts of $f, g$. Then we have

$$
R T(f, \tilde{f}, g, \tilde{g} ; U)=\frac{1}{\left[\Pi_{1}: \Gamma_{1}\right]} \sum_{\bar{\beta} \in \Pi_{2} / \Gamma_{2}} \rho_{\beta} \circ \hat{i}_{2}^{\beta}(R T(\bar{\beta} \bar{f}, \beta \tilde{f}, \bar{g}, \tilde{g} ; \bar{U})),
$$

where $\beta \in \Pi_{2}$ is any element whose image in $\Pi_{2} / \Gamma_{2}$ is $\bar{\beta}$.

Proof. We will follow the proof of Theorem 4.2. Throughout the proof, for $U \subset M_{1}$, let $\bar{U}=\bar{p}_{1}^{-1}(U) \subset \bar{M}_{1}$. For any tuple $(f, \tilde{f}, g, \tilde{g}) \in \mathcal{C}\left(M_{1}, M_{2}\right)$, let $\bar{f}, \bar{g}: \bar{M}_{1} \rightarrow \bar{M}_{2}$ be given by the diagram (2-5). Let:

$$
\sigma(f, \tilde{f}, g, \tilde{g} ; U)=\frac{1}{\left[\Pi_{1}: \Gamma_{1}\right]} \sum_{\bar{\beta} \in \Pi_{2} / \Gamma_{2}} \rho_{\beta} \circ \hat{i}_{2}^{\beta}(R T(\bar{\beta} \bar{f}, \beta \tilde{f}, \bar{g}, \tilde{g} ; \bar{U})),
$$

where $\bar{f}, \bar{g}$ are any fixed lifts of $f$ and $g$, and we will show that $\sigma$ satisfies the 5 axioms of Theorem 4.3 .

First we show that $\sigma$ is well defined, that is, independent of the element $\beta \in \Pi_{2}$ which projects to $\bar{\beta} \in \Pi_{2} / \Gamma_{2}$. It suffices to show that if $\beta$ and $\beta^{\prime}$ each project to $\bar{\beta}$, then

$$
\rho_{\beta} \circ \hat{i}_{2}^{\beta}(R T(\bar{\beta} \bar{f}, \beta \tilde{f}, \bar{g}, \tilde{g} ; \bar{U}))=\rho_{\beta^{\prime}} \circ \hat{i}_{2}^{\beta^{\prime}}\left(R T\left(\bar{\beta} \bar{f}, \beta^{\prime} \tilde{f}, \bar{g}, \tilde{g} ; \bar{U}\right)\right) .
$$

Let $\gamma \in \Gamma_{2}$ be some element with $\gamma \beta=\beta^{\prime}$. Then we must show

$$
\rho_{\beta} \circ \hat{i}_{2}^{\beta}(R T(\bar{\beta} \bar{f}, \beta \tilde{f}, \bar{g}, \tilde{g} ; \bar{U}))=\rho_{\gamma \beta} \circ \hat{i}_{2}^{\gamma \beta}(R T(\bar{\beta} \bar{f},(\gamma \beta) \tilde{f}, \bar{g}, \tilde{g} ; \bar{U})),
$$

This follows from the following observation: For $\beta \in \Pi_{2}$ and $\gamma \in \Gamma_{2}$, we have the following commutative diagram

$$
\begin{array}{ccc}
\rho_{\gamma \beta} \circ \hat{\iota}_{2}^{\gamma \beta}=\rho_{\beta} \circ \hat{\iota}_{2}^{\beta} \circ \rho_{\gamma} & \\
\mathcal{R}\left[\tau_{\gamma \beta} \phi^{\prime}, \psi^{\prime}\right] & \stackrel{\hat{\iota}_{2}^{\gamma \beta}}{\longrightarrow} \mathcal{R}\left[\tau_{\gamma \beta} \phi, \psi\right] \stackrel{\rho_{\gamma \beta}}{\longrightarrow} & \mathcal{R}[\phi, \psi] \\
\downarrow & & \uparrow \rho_{\beta} \\
\mathcal{R}\left[\tau_{\gamma}\left(\tau_{\beta} \phi\right)^{\prime}, \psi^{\prime}\right] \stackrel{\rho_{\gamma}}{\longrightarrow} \mathcal{R}\left[\tau_{\beta} \phi^{\prime}, \psi^{\prime}\right] \stackrel{\hat{\iota}_{2}^{\beta}}{\longrightarrow} \mathcal{R}\left[\tau_{\beta} \phi, \psi\right]
\end{array}
$$




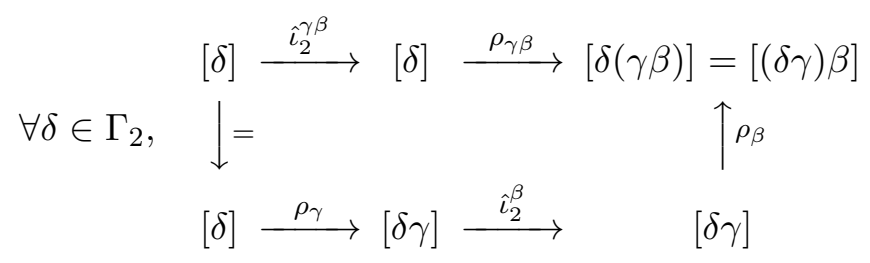

Then we have

$$
\begin{aligned}
\rho_{\gamma \beta} \circ \hat{i}_{2}^{\gamma \beta}(R T(\bar{\beta} \bar{f},(\gamma \beta) \tilde{f}, \bar{g}, \tilde{g} ; \bar{U})) & =\rho_{\beta} \circ \hat{i}_{2}^{\beta} \circ \rho_{\gamma}(R T(\bar{\beta} \bar{f},(\gamma \beta) \tilde{f}, \bar{g}, \tilde{g} ; \bar{U})) \\
& =\rho_{\beta} \circ \hat{i}_{2}^{\beta}(R T(\bar{\beta} \bar{f}, \beta \tilde{f}, \bar{g}, \tilde{g} ; \bar{U})),
\end{aligned}
$$

as required, where the last equality is by $(2-3)$, which holds for the local coincidence Reidemeister trace (this is [13, Theorem 6]).

Now we verify that our five axioms hold for $\sigma$. The homotopy axiom is satisfied exactly as in Theorem 4.2. Any pair of admissible homotopies $(H, G)$ from $f$ to $f^{\prime}$ and $g$ to $g^{\prime}$ will uniquely determine a pair of admissible homotopies $(\bar{\beta} \bar{H}, \bar{G})$ from $\bar{\beta} \bar{f}$ to $\bar{\beta} \bar{f}^{\prime}$ and $\bar{g}$ to $\bar{g}^{\prime}$, and also a pair of admissible homotopies from $\tilde{f}$ to $\tilde{f}^{\prime}$ and $\tilde{g}$ to $\tilde{g}^{\prime}$. Then by the homotopy property for the Reidemeister trace we will have:

$$
\begin{aligned}
\sigma(f, \tilde{f}, g, \tilde{g} ; U) & =\frac{1}{\left[\Pi_{1}: \Gamma_{1}\right]} \sum_{\bar{\beta} \in \Pi_{2} / \Gamma_{2}} \rho_{\beta} \circ \hat{i}_{2}^{\beta}(R T(\bar{\beta} \bar{f}, \beta \tilde{f}, \bar{g}, \tilde{g} ; \bar{U})) \\
& =\frac{1}{\left[\Pi_{1}: \Gamma_{1}\right]} \sum_{\bar{\beta} \in \Pi_{2} / \Gamma_{2}} \rho_{\beta} \circ \hat{i}_{2}^{\beta}\left(R T\left(\bar{\beta} \bar{f}^{\prime}, \beta \tilde{f}^{\prime}, \bar{g}^{\prime}, \tilde{g}^{\prime} ; \bar{U}\right)\right) \\
& =\sigma\left(f^{\prime}, \tilde{f}^{\prime}, g^{\prime}, \tilde{g}^{\prime} ; U\right)
\end{aligned}
$$

For the additivity axiom again we follow exactly the argument from Theorem 4.2, We omit the details.

For the normalization axiom, let $c$ be a constant map and $g$ be an orientation preserving embedding of some small set $U$ with $g(x)=c$ for some $x \in U$. Then there is some $\bar{\alpha} \in \Pi_{2} / \Gamma_{2}$ with $\bar{g}(\bar{x})=\bar{\alpha} \bar{c}$.

We will assume that $U$ is sufficiently small so that $\bar{U}$ is a union of finitely many components homeomorphic to $U$ by $p_{1}$. Let $\bar{U}_{0}$ be the connected subset of $\bar{U}$ which contains $\bar{x}$. Then we have a disjoint union

$$
\bar{U}=\bigsqcup_{\bar{\gamma} \in \Pi_{1} / \Gamma_{1}} \bar{\gamma}\left(\bar{U}_{0}\right)
$$


and

$$
\begin{aligned}
\epsilon(\sigma(c, \tilde{c}, g, \tilde{g} ; U)) & =\epsilon\left(\frac{1}{\left[\Pi_{1}: \Gamma_{1}\right]} \sum_{\bar{\beta} \in \Pi_{2} / \Gamma_{2}} \rho_{\beta} \circ \hat{i}_{2}^{\beta}(R T(\bar{\beta} \bar{c}, \beta \tilde{c}, \bar{g}, \tilde{g} ; \bar{U}))\right) \\
& =\frac{1}{\left[\Pi_{1}: \Gamma_{1}\right]} \sum_{\bar{\beta} \in \Pi_{2} / \Gamma_{2}} \epsilon(R T(\bar{\beta} \bar{c}, \beta \tilde{c}, \bar{g}, \tilde{g} ; \bar{U})) \\
& =\frac{1}{\left[\Pi_{1}: \Gamma_{1}\right]} \sum_{\bar{\beta} \in \Pi_{2} / \Gamma_{2}} \sum_{\bar{\gamma} \in \Pi_{1} / \Gamma_{1}} \epsilon\left(R T\left(\bar{\beta} \bar{c}, \beta \tilde{c}, \bar{g}, \tilde{g} ; \bar{\gamma}\left(\bar{U}_{0}\right)\right)\right)
\end{aligned}
$$

The set $\operatorname{Coin}(\bar{\beta} \bar{c}, \bar{g}) \cap \bar{\gamma}\left(\bar{U}_{0}\right)$ is nonempty when $\bar{g}(\bar{\gamma} \bar{x})=\bar{\beta} \bar{c}$. Since $\bar{g}(\bar{x})=$ $\bar{\alpha} \bar{c}$, this is equivalent to $\bar{\psi}(\bar{\gamma}) \bar{g}(\bar{x})=\bar{\beta} \bar{\alpha}^{-1} \bar{g}(\bar{x})$, which is to say that $\bar{\psi}(\bar{\gamma})=$ $\bar{\beta} \bar{\alpha}^{-1}$.

In the case where $\operatorname{Coin}(\bar{\beta} \bar{c}, \bar{g}) \cap \bar{\gamma}\left(\bar{U}_{0}\right)$ is nonempty, it contains a single coincidence point $\bar{\gamma} \bar{x}$, and $\bar{g}$ is an orientation preserving embedding of $\bar{\gamma}\left(\bar{U}_{0}\right)$, because covering transformations of covering spaces of orientable manifolds are orientation preserving. Thus by the normalization property for $R T$ we have:

$$
\epsilon\left(R T\left(\bar{\beta} \bar{c}, \beta \tilde{c}, \bar{g}, \tilde{g} ; \bar{\gamma}\left(\bar{U}_{0}\right)\right)\right)= \begin{cases}1 & \text { if } \bar{\psi}(\bar{\gamma})=\bar{\beta} \bar{\alpha}^{-1} \\ 0 & \text { otherwise }\end{cases}
$$

Then the above summation gives:

$$
\begin{aligned}
\epsilon(\sigma(c, \tilde{c}, g, \tilde{g} ; U)) & =\frac{1}{\left[\Pi_{1}: \Gamma_{1}\right]} \sum_{\bar{\beta} \in \Pi_{2} / \Gamma_{2}} \#\left\{\bar{\gamma} \in \Pi_{1} / \Gamma_{1} \mid \bar{\psi}(\bar{\gamma})=\bar{\beta} \bar{\alpha}^{-1}\right\} \\
& =\frac{1}{\left[\Pi_{1}: \Gamma_{1}\right]} \sum_{\bar{\beta} \in \Pi_{2} / \Gamma_{2}} \# \bar{\psi}^{-1}\left(\left\{\bar{\beta} \bar{\alpha}^{-1}\right\}\right) \\
& =\frac{1}{\left[\Pi_{1}: \Gamma_{1}\right]} \sum_{\bar{\delta} \in \Pi_{2} / \Gamma_{2}} \# \bar{\psi}^{-1}(\{\delta\}) .
\end{aligned}
$$

The set inside the sum has 0 elements when $\delta \in \operatorname{im} \bar{\psi}$, and otherwise has size \# ker $\bar{\psi}$. Thus we have:

$$
\epsilon(\sigma(c, \tilde{c}, g, \tilde{g} ; U))=\frac{1}{\left[\Pi_{1}: \Gamma_{1}\right]} \# \operatorname{im} \bar{\psi} \cdot \# \operatorname{ker} \bar{\psi}=1
$$

by the First Isomorphism Theorem. 
For the lift invariance axiom, we have

$$
\begin{aligned}
\epsilon(\sigma(f, \alpha \tilde{f}, g, \gamma \tilde{g} ; U)) & =\epsilon\left(\frac{1}{\left[\Pi_{1}: \Gamma_{1}\right]} \sum_{\bar{\beta} \in \Pi_{2} / \Gamma_{2}} \rho_{\beta} \circ \hat{i}_{2}^{\beta}(R T(\bar{\beta} \bar{f}, \beta \alpha \tilde{f}, \bar{g}, \gamma \tilde{g} ; \bar{U}))\right) \\
& =\frac{1}{\left[\Pi_{1}: \Gamma_{1}\right]} \sum_{\bar{\beta} \in \Pi_{2} / \Gamma_{2}} \epsilon(R T(\bar{\beta} \bar{f}, \beta \alpha \tilde{f}, \bar{g}, \gamma \tilde{g} ; \bar{U})) \\
& =\frac{1}{\left[\Pi_{1}: \Gamma_{1}\right]} \sum_{\bar{\beta} \in \Pi_{2} / \Gamma_{2}} \epsilon(R T(\bar{\beta} \bar{f}, \beta \tilde{f}, \bar{g}, \tilde{g} ; \bar{U})) \\
& =\epsilon(\sigma(f, \tilde{f}, g, \tilde{g} ; U)) .
\end{aligned}
$$

Finally we must demonstrate the coincidence of lifts axiom. Say that $[\alpha]$ appears in $\sigma(f, \tilde{f}, g, \tilde{g} ; U)$ with nonzero coefficient, and we must show that $\alpha \tilde{f}$ and $\tilde{g}$ have a coincidence in $p_{1}^{-1}(U)$. By (4-2), if $[\alpha]$ has nonzero coefficient in $\sigma(f, \tilde{f}, g, \tilde{g} ; U)$, then there is some $\bar{\beta}$ and some $\gamma$ such that $\rho_{\beta}\left(\hat{i}_{2}^{\beta}([\gamma])\right)=[\alpha]$, and $[\gamma]$ has nonzero coefficient in $R T(\bar{\beta} \bar{f}, \beta \tilde{f}, \bar{g}, \tilde{g} ; \bar{U})$.

Then by the coincidence of lifts property applied to $R T(\bar{\beta} \bar{f}, \beta \tilde{f}, \bar{g}, \tilde{g} ; \bar{U})$, the maps $\gamma \beta \tilde{f}$ and $\tilde{g}$ have a coincidence on $\bar{U}$. But since $\rho_{\beta}\left(\hat{i}_{2}^{\beta}([\gamma])\right)=[\alpha]$, we have $\alpha=\gamma \beta$, and thus $\alpha \tilde{f}$ and $\tilde{g}$ have a coincidence on $\bar{U}$ as desired.

We have shown that $\sigma$ satisfies the 5 axioms of Theorem 4.3, and thus it must equal the coincidence Reidmemeister trace.

\section{EXAmPles}

Example 5.1. Let $M_{1}$ be a 3-dimensional orientable flat manifold $\Pi_{1} \backslash \mathbb{R}^{3}$ where $\Pi_{1}$ is the 3 -dimensional orientable Bieberbach group $\mathfrak{G}_{2}$ [14, Theorem 3.5.5]:

$$
\Pi_{1}=\left\langle t_{1}, t_{2}, t_{3}, \alpha \mid\left[t_{i}, t_{j}\right]=1, \alpha^{2}=t_{1}, \alpha t_{2} \alpha^{-1}=t_{2}^{-1}, \alpha t_{3} \alpha^{1}=t_{3}^{-1}\right\rangle .
$$

We can embed this group into $\operatorname{Aff}\left(\mathbb{R}^{3}\right)$ by taking $\left\{\mathbf{e}_{1}, \mathbf{e}_{2}, \mathbf{e}_{3}\right\}$ as the standard basis for $\mathbb{R}^{3}$ and

$$
t_{i}=\left(\mathbf{e}_{i}, I_{3}\right)(i=1,2,3), \quad \alpha=\left(\left(\begin{array}{c}
\frac{1}{2} \\
0 \\
0
\end{array}\right),\left(\begin{array}{rrr}
1 & 0 & 0 \\
0 & -1 & 0 \\
0 & 0 & -1
\end{array}\right)\right) .
$$

Then the translation lattice is

$$
\Gamma_{1}=\Pi_{1} \cap \mathbb{R}^{3}=\left\langle t_{1}, t_{2}, t_{3}\right\rangle=\left\langle\mathbf{e}_{1}, \mathbf{e}_{2}, \mathbf{e}_{3}\right\rangle=\mathbb{Z}^{3}
$$

and the holonomy group is $\Pi_{1} / \Gamma_{1} \cong \mathbb{Z}_{2}$. 
Let $M_{2}=\mathbb{R} P^{3}$. Assume that there is a commutative diagram

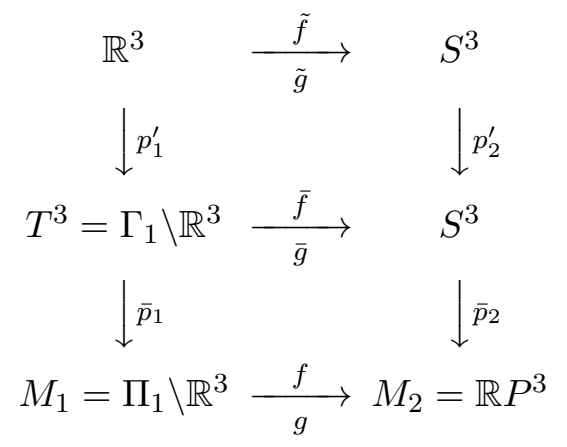

inducing the following commutative diagram

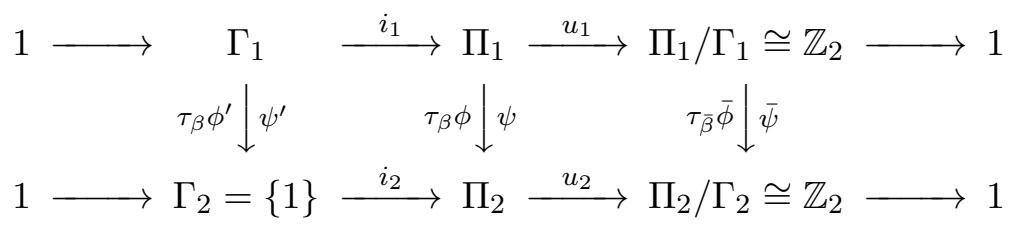

where $\bar{\phi}$ is an isomorphism and $\bar{\psi}$ is a trivial homomorphism.

Let $\beta$ be the antipodal map of $S^{3}$. Then $\Pi_{2}=\langle\beta\rangle$. Since $\Pi_{2}$ is abelian, we have $\tau_{\beta} \phi=\phi$ and $\tau_{\bar{\beta}} \bar{\phi}=\bar{\phi}$.

Remark that since $\psi^{\prime}$ and $\bar{\psi}$ are trivial homomorphisms, it follows that $\psi$ is a trivial homomorphism. Since $\phi^{\prime}$ is a trivial homomorphism and $\bar{\phi}$ is an isomorphism, it follows that $\phi(\alpha)=\beta$.

A simple computation shows that

$$
\begin{aligned}
1 & \rightarrow \operatorname{coin}\left(\phi^{\prime}, \psi^{\prime}\right)=\Gamma_{1} \rightarrow \operatorname{coin}(\phi, \psi)=\Gamma_{1} \rightarrow \operatorname{coin}(\bar{\phi}, \bar{\psi})=\{\overline{1}\} \\
& \rightarrow \mathcal{R}\left[\phi^{\prime}, \psi^{\prime}\right]=\{[1]\} \rightarrow \mathcal{R}[\phi, \psi]=\{[1]=[\beta]\} \rightarrow \mathcal{R}[\bar{\phi}, \bar{\psi}]=\{[\overline{1}]=[\bar{\beta}]\} \rightarrow 1
\end{aligned}
$$

There is only one coincidence class of $(f, g)$ :

$$
\mathbb{S}_{[1]}=p_{1}(\operatorname{Coin}(\tilde{f}, \tilde{g})) \text {. }
$$

Hence

$$
R T(f, \tilde{f}, g, \tilde{g})=\operatorname{ind}\left(f, g ; \mathbb{S}_{[1]}\right)[1] .
$$

On the other hand, because $\tau_{\beta} \phi=\phi$, we have

$$
R T(\bar{\beta} \bar{f}, \beta \tilde{f}, \bar{g}, \tilde{g})=R T(\bar{f}, \tilde{f}, \bar{g}, \tilde{g})=\operatorname{ind}\left(\bar{f}, \bar{g} ; \mathbb{S}_{[1]}\right)[1] \in \mathbb{Z} \mathcal{R}\left[\phi^{\prime}, \psi^{\prime}\right] .
$$

Thus

$$
\begin{aligned}
\frac{1}{\left[\Pi_{1}: \Gamma_{1}\right]} & \sum_{\bar{\beta} \in \Pi_{2} / \Gamma_{2}} \rho_{\beta} \circ \hat{i}^{\beta}(R T(\bar{\beta} \bar{f}, \beta \tilde{f}, \bar{g}, \tilde{g})) \\
= & \frac{1}{2}\left(\rho_{1} \circ \hat{i}_{2}^{1}(R T(\bar{f}, \tilde{f}, \bar{g}, \tilde{g}))+\rho_{\beta} \circ \hat{i}_{2}^{\beta}(R T(\bar{\beta} \bar{f}, \beta \tilde{f}, \bar{g}, \tilde{g}))\right) \\
= & \frac{1}{2}\left(\rho_{1} \circ \hat{i}_{2}^{1}\left(\operatorname{ind}\left(\bar{f}, \bar{g} ; \mathbb{S}_{[1]}\right)[1]\right)+\rho_{\beta} \circ \hat{i}_{2}^{\beta}\left(\operatorname{ind}\left(\bar{f}, \bar{g} ; \mathbb{S}_{[1]}\right)[1]\right)\right) \\
= & \operatorname{ind}\left(f, g ; \mathbb{S}_{[1]}\right)[1] .
\end{aligned}
$$


This example demonstrates how the averaging formula for the coincidence Reidemeister trace holds. Notice also in this example that the averaging formula for the Nielsen coincidence number also holds. This is expected from from [8, Theorem 4.6] since $\operatorname{coin}(\phi, \psi) \subset \Gamma_{1}$.

Example 5.2. Let $M_{1}=\mathbb{R} P^{3}$. Let $M_{2}$ be a 3-dimensional orientable flat manifold $\Pi_{2} \backslash \mathbb{R}^{3}$ where $\Pi_{2}$ is the 3 -dimensional orientable Bieberbach group $\mathfrak{G}_{2}$ :

$$
\Pi_{2}=\left\langle t_{1}, t_{2}, t_{3}, \alpha \mid\left[t_{i}, t_{j}\right]=1, \alpha^{2}=t_{1}, \alpha t_{2} \alpha^{-1}=t_{2}^{-1}, \alpha t_{3} \alpha^{1}=t_{3}^{-1}\right\rangle .
$$

Let $\Gamma_{2}=\left\langle t_{1}, t_{2}, t_{3}\right\rangle=\mathbb{Z}^{3}$.

Assume that there is a commutative diagram

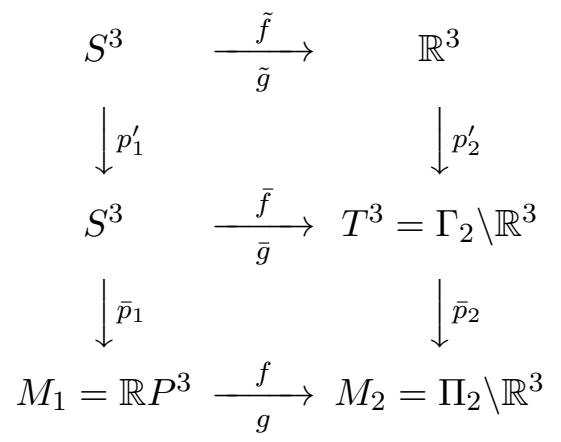

inducing the following commutative diagram

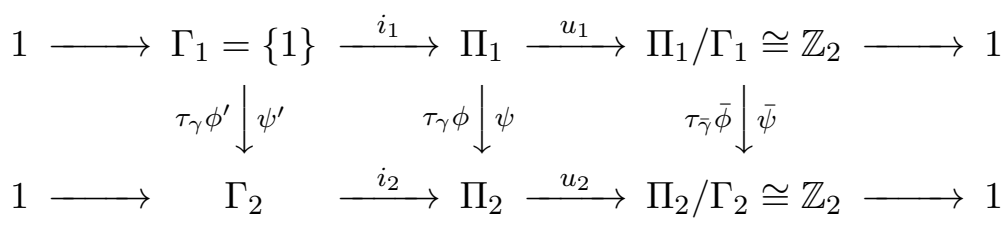

Let $\beta$ be the antipodal map of $S^{3}$. Then $\Pi_{1}=\langle\beta\rangle$. Notice that every homomorphism $\xi: \Pi_{1} \rightarrow \Pi_{2}$ must be a trivial homomorphism. Otherwise, $\xi(\beta)$ is of the form

$$
\xi(\beta)=t_{1}^{n_{1}} t_{2}^{n_{2}} t_{3}^{n_{3}} \alpha
$$

Then $1=\xi\left(\beta^{2}\right)=\xi(\beta)^{2}=\left(t_{1}^{n_{1}} t_{2}^{n_{2}} t_{3}^{n_{3}} \alpha\right)^{2}=t_{1}^{2 n_{1}+1}$, which is impossible. Thus $\tau_{\gamma} \phi$ and $\psi$ are trivial, and the remaining homomorphisms are also trivial.

A simple computation shows that

$$
\begin{aligned}
1 & \rightarrow \operatorname{coin}\left(\phi^{\prime}, \psi^{\prime}\right)=\Gamma_{1} \rightarrow \operatorname{coin}(\phi, \psi)=\Pi_{1} \rightarrow \operatorname{coin}(\bar{\phi}, \bar{\psi})=\Pi_{1} / \Gamma_{1} \\
& \rightarrow \mathcal{R}\left[\phi^{\prime}, \psi^{\prime}\right]=\Gamma_{2} \rightarrow \mathcal{R}[\phi, \psi]=\Pi_{2} \rightarrow \mathcal{R}[\bar{\phi}, \bar{\psi}]=\Pi_{2} / \Gamma_{2} \rightarrow 1 .
\end{aligned}
$$

The coincidence classes of $(f, g)$ are

$$
\mathbb{S}_{\gamma}=p_{1}(\operatorname{Coin}(\gamma \tilde{f}, \tilde{g})), \quad \forall \gamma \in \Pi_{2} .
$$

Hence

$$
R T(f, \tilde{f}, g, \tilde{g})=\sum_{\gamma \in \Pi_{2}} \operatorname{ind}\left(f, g ; \mathbb{S}_{\gamma}\right) \gamma \in \mathbb{Z} \Pi_{2}
$$


On the other hand,

$$
\begin{aligned}
\frac{1}{\left[\Pi_{1}: \Gamma_{1}\right]} & \sum_{\bar{\gamma} \in \Pi_{2} / \Gamma_{2}} \rho_{\gamma} \circ \hat{i}^{\gamma}(R T(\bar{\gamma} \bar{f}, \gamma \tilde{f}, \bar{g}, \tilde{g})) \\
= & \frac{1}{2}\left(\rho_{1} \circ \hat{i}_{2}^{1}(R T(\bar{f}, \tilde{f}, \bar{g}, \tilde{g}))+\rho_{\alpha} \circ \hat{i}_{2}^{\alpha}(R T(\bar{\alpha} \bar{f}, \alpha \tilde{f}, \bar{g}, \tilde{g}))\right) \\
= & \frac{1}{2}\left(\rho_{1} \circ \hat{i}_{2}^{1}\left(\sum_{\gamma \in \Gamma_{2}} \operatorname{ind}\left(\bar{f}, \bar{g} ; \mathbb{S}_{\gamma}\right) \gamma\right)+\rho_{\alpha} \circ \hat{i}_{2}^{\alpha}\left(\sum_{\gamma \in \Gamma_{2}} \operatorname{ind}\left(\bar{\alpha} \bar{f}, \bar{g} ; \mathbb{S}_{\gamma}\right) \gamma\right)\right) \\
= & \sum_{\gamma \in \Gamma_{2}} \frac{1}{2} \operatorname{ind}\left(\bar{f}, \bar{g} ; p_{1}^{\prime}(\operatorname{Coin}(\gamma \tilde{f}, \tilde{g})) \gamma+\sum_{\gamma \in \Gamma_{2}} \frac{1}{2} \operatorname{ind}\left(\bar{\alpha} \bar{f}, \bar{g} ; p_{1}^{\prime}(\operatorname{Coin}(\gamma \alpha \tilde{f}, \tilde{g})) \gamma \alpha\right.\right. \\
= & \sum_{\gamma \in \Gamma_{2}} \operatorname{ind}\left(f, g ; p_{1}(\operatorname{Coin}(\gamma \tilde{f}, \tilde{g})) \gamma+\sum_{\gamma \in \Gamma_{2}} \operatorname{ind}\left(f, g ; p_{1}(\operatorname{Coin}(\gamma \alpha \tilde{f}, \tilde{g})) \gamma \alpha .\right.\right.
\end{aligned}
$$

Consequently, we have

$$
R T(f, \tilde{f}, g, \tilde{g})=\frac{1}{\left[\Pi_{1}: \Gamma_{1}\right]} \sum_{\bar{\gamma} \in \Pi_{2} / \Gamma_{2}} \rho_{\gamma} \circ \hat{i}^{\gamma}(R T(\bar{\gamma} \bar{f}, \gamma \tilde{f})) .
$$

\section{Open questions in the nONORIEntable CASE}

Nielsen coincidence theory for nonorientable manifolds of the same dimension is generally defined in terms of the "semi-index" of Dobrenko and Jezierski [1]. We denote the semi-index of a coincidence class $\mathbb{S}$ by $\mid$ ind $\mid(f, g ; \mathbb{S})$. The semi-index can be used to define a coincidence Reidemeister trace as in $(2-2)$.

$$
|R T|(f, \tilde{f}, g, \tilde{g})=\sum_{[\beta] \in \mathcal{R}[\phi, \psi]}|\operatorname{ind}|\left(f, g ; \mathbb{S}_{[\beta]}\right)[\beta] \in \mathbb{Z} \mathcal{R}[\phi, \psi] .
$$

When the manifolds $M_{1}$ and $M_{2}$ are orientable, it is well known that $\mid$ ind $|(f, g ; \mathbb{S})=| \operatorname{ind}(f, g ; \mathbb{S}) \mid$, the absolute value of the classical coincidence index. By the definition of $|R T|$, we will have the similar formula

$$
|R T|(f, \tilde{f}, g, \tilde{g})=|R T(f, \tilde{f}, g, \tilde{g})|,
$$

where the absolute values on the right denote taking absolute values of each integer coefficient, after grouping together all terms.

It is natural to ask whether an averaging formula will hold for this semiindex Reidemeister trace.

Question. Does the following formula hold?

$$
|R T|(f, \tilde{f}, g, \tilde{g})=\frac{1}{\left[\Pi_{1}: \Gamma_{1}\right]} \sum_{\bar{\beta} \in \Pi_{2} / \Gamma_{2}} \rho_{\beta} \circ \hat{i}_{2}^{\beta}(|R T|(\bar{\beta} \bar{f}, \beta \tilde{f}, \bar{g}, \tilde{g}))
$$

This formula will hold when the manifolds are orientable- it is not clear if the formula holds when the manifolds are nonorientable. In the following example, the averaging formula does hold. 
Example 6.1. Consider

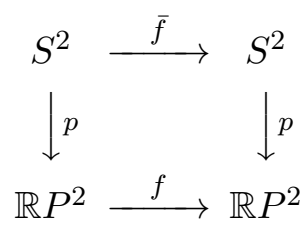

with $f=\operatorname{id}_{\mathbb{R} P^{2}}$ and $\tilde{f}=\operatorname{id}_{S^{2}}$. Then the diagram induces the identity endomorphism $\phi$ of the group $\Pi=\{1, \alpha\}$ of transformations of $p$. Observe that

- $\mathcal{R}[\phi, \phi]=\{[1],[\alpha]\}$,

- $p(\operatorname{Coin}(\tilde{f}, \tilde{f}))=\mathbb{R} P^{2}$ and $p(\operatorname{Coin}(\alpha \tilde{f}, \tilde{f}))=\emptyset$,

- $|\operatorname{ind}|(f, f ; p(\operatorname{Coin}(\tilde{f}, \tilde{f})))=1$

(by changing one $f$ to be a small rotation), and |ind $\mid(f, f ; p(\operatorname{Coin}(\alpha \tilde{f}, \tilde{f})))=0$.

Then we have

$$
\begin{aligned}
|R T|(f, \tilde{f}, f, \tilde{f}) & =|\operatorname{ind}|(f, f ; p(\operatorname{Coin}(\tilde{f}, \tilde{f})))[1]+|\operatorname{ind}|(f, f ; p(\operatorname{Coin}(\alpha \tilde{f}, \tilde{f})))[\alpha] \\
& =1[1]+0[\alpha]=1[1] \in \mathbb{Z} \mathcal{R}[\phi, \phi], \\
N(f, f) & =1 .
\end{aligned}
$$

On the other hand we can compute $|R T|(\tilde{f}, \tilde{f}, \tilde{f}, \tilde{f})$ and $|R T|(\alpha \tilde{f}, \alpha \tilde{f}, \tilde{f}, \tilde{f})$. Because the manifold involved is an orientable manifold $S^{2},|R T|$ is the absolute value of the classical $R T$. For the computation, we remark that $\Gamma=\{1\}$ and so $\phi^{\prime}$ is trivial, hence $\bar{\Pi}=\Pi$ and $\bar{\phi}=\phi$. Indeed, we have

$$
\begin{aligned}
& |R T|(\tilde{f}, \tilde{f}, \tilde{f}, \tilde{f})=\left|\operatorname{ind}\left(\tilde{f}, \tilde{f} ; S^{2}\right)\right|[1]=2[1] \in \mathbb{Z} \mathcal{R}\left[\phi^{\prime}, \phi^{\prime}\right], \\
& |R T|(\alpha \tilde{f}, \alpha \tilde{f}, \tilde{f}, \tilde{f})=|\operatorname{ind}(\alpha \tilde{f}, \tilde{f} ; \emptyset)|[1]=0[1] \in \mathbb{Z} \mathcal{R}\left[\tau_{\alpha} \phi^{\prime}, \phi^{\prime}\right],
\end{aligned}
$$

hence

$\frac{1}{2}\left\{\rho_{1} \circ \hat{\iota}_{2}^{1}(|R T|(\tilde{f}, \tilde{f}, \tilde{f}, \tilde{f}))+\rho_{\alpha} \circ \hat{\iota}_{2}^{\alpha}(|R T|(\alpha \tilde{f}, \alpha \tilde{f}, \tilde{f}, \tilde{f}))\right\}=\frac{1}{2}\{2[1]+0[1]\}$.

Consequently, we have

$|R T|(f, \tilde{f}, f, \tilde{f})=\frac{1}{2}\left\{\rho_{1} \circ \hat{\iota}_{2}^{1}(|R T|(\tilde{f}, \tilde{f}, \tilde{f}, \tilde{f}))+\rho_{\alpha} \circ \hat{\iota}_{2}^{\alpha}(|R T|(\alpha \tilde{f}, \alpha \tilde{f}, \tilde{f}, \tilde{f}))\right\}$.

Note that in this example the semi-index Nielsen number does not average:

$$
N(f, f)=1 \neq \frac{1}{2}(1+0)=\frac{1}{2}(N(\tilde{f}, \tilde{f})+N(\alpha \tilde{f}, \tilde{f})) .
$$

This was expected already by [8, Theorem 4.5] as $\operatorname{coin}(\phi, \phi)=\Pi \not \subset \Gamma$.

The real issue in deriving an averaging formula for the semi-index Reidemeister trace seems to be the semi-index itself. The following question seems likely to be easier, but we still do not have an answer: 
Question. When $M_{1}$ and $M_{2}$ are possibly nonorientable, does the following formula hold?

$$
\mid \text { ind }\left|(f, g ; \mathbb{S})=\frac{1}{\left[\Pi_{1}: \Gamma_{1}\right]} \sum_{\bar{\beta} \in \Pi_{2} / \Gamma_{2}}\right| \operatorname{ind} \mid(\bar{\beta} f, \bar{g} ; \overline{\mathbb{S}})
$$

The axiomatic approach is not likely to succeed in answering these questions, since there is no axiomatic formulation of the semi-index. In particular the semi-index is defined only for a coincidence class, not an arbitrary (compact) coincidence set. Therefore the semi-index will not have an additivity property like the one used in $[2,12,13,3$.

A nonorientable version of Theorem 4.3 is proved in [3] using an index with values in $\mathbb{Z} \oplus \mathbb{Z}_{2}$ which is closely related to the semi-index. Roughly, the $\mathbb{Z}$-part of the index is analogous to the classical index of nondegenerate coincidence points, while the $\mathbb{Z}_{2}$-part carries information about any degenerate coincidences. The $\mathbb{Z} \oplus \mathbb{Z}_{2}$-index of a coincidence class is zero if and only if the semi-index is zero, so a Nielsen number using the $\mathbb{Z} \oplus \mathbb{Z}_{2}$-index will agree with the traditional semi-index Nielsen number. Unlike the semiindex, the $\mathbb{Z} \oplus \mathbb{Z}_{2}$ index can be defined for any coincidence set, and has the appropriate additivity property.

The fact that the values are not integers introduces new complications for a formulation of an averaging formula like (6-1) for the $\mathbb{Z} \oplus \mathbb{Z}_{2}$-index. In particular the factor of $1 /\left[\Pi_{1}: \Gamma_{1}\right]$ needs to be reinterpreted if the sum gives a non-integer value, and also some adjustment must be made for the fact that when the covering spaces are all orientable, the index of all lifts

will have values in $\mathbb{Z}$, while the index of the maps themselves may still have nontrivial $\mathbb{Z}_{2}$ part.

Still, the axioms for the $\mathbb{Z} \oplus \mathbb{Z}_{2}$-index may provide a simple way to prove an averaging formula, if it can be properly formulated. Therefore we ask:

Question. Is there an averaging formula for the $\mathbb{Z} \oplus \mathbb{Z}_{2}$-index analogous to (6-1)?

\section{REFERENCES}

[1] R. Dobreńko and J. Jezierski, The coincidence Nielsen number on nonorientable manifolds, Rocky Mountain J. Math., 23 (1993), 67-85.

[2] M. Furi, M. P. Pera and M. Spadini, On the uniqueness of the fixed point index on differentiable manifolds, Fixed Point Theory Appl., 2004:4, 251-259.

[3] D. Gonçalves and P. C. Staecker, Axioms for the coincidence index of maps between manifolds of the same dimension, Topology Appl., 159 (2012), 3760-3776.

[4] K. Y. Ha, J. B. Lee and P. Penninckx, Formulas for the Reidemeister, Lefschetz and Nielsen coincidence numbers of maps between infra-nilmanifolds, Fixed Point Theory Appl., 2012:39 (2012), 1-23.

[5] J. Jezierski, The Nielsen coincidence theory for topological manifolds, Fundamenta Mathematicae 143 (1993), 167-178.

[6] B. Jiang, Nielsen Fixed Point Theory, Contemporary Mathematics, vol. 14, American Mathematical Society, Providence, R.I., 1983.

[7] S. W. Kim, J. B. Lee and K. B. Lee, Averaging formula for Nielsen numbers, Nagoya Math. J., 178 (2005), 37-53. 
[8] S. W. Kim and J. B. Lee, Averaging formula for Nielsen coincidence numbers, Nagoya Math. J., 186 (2007), 69-93.

[9] J. Liu and X. Zhao, An averaging formula for Reidemeister traces, Topol. Methods Nonlinear Anal., 46 (2015), 17-27.

[10] C. McCord, Estimating Nielsen numbers on infrasolvmanifolds, Pacific J. Math., 154 (1992), 345-368.

[11] C. McCord, Lefschetz and Nielsen coincidence numbers on nilmanifolds and solvmanifolds II, Topology Appl., 75 (1997), 81-?92.

[12] P. C. Staecker, On the uniqueness of the coincidence index on orientable differentiable manifolds, Topology Appl., 154 (2007), 1961-1970. arxiv eprint math.GN/0607751

[13] P. C. Staecker, Axioms for a local Reidemeister trace in fixed point and coincidence theory on differentiable manifolds, J. Fixed Point Theory Appl., 5 (2009), 237-247. arxiv eprint 0704.1891.

[14] J. Wolf, Spaces of constant curvature, 5th edition, Publish or Perish, Inc., Houston, TX, 1984.

Department of Mathematics, Sogang University, Seoul 04107, KOREA

E-mail address: jlee@sogang.ac.kr

Department of Mathematics, Fairfield University, Fairfield CT, 06824, USA

E-mail address: cstaecker@fairfield.edu 